\title{
Alterstice
}

Revue internationale de la recherche interculturelle

International Journal of Intercultural Research

Revista International de la Investigacion Intercultural

\section{Psychologies et cultures, sous la direction de B. Troadec et T. Bellaj}

\section{Pierre R. Dasen}

Volume 1, numéro 2, 2011

URI : https://id.erudit.org/iderudit/1077609ar

DOI : https://doi.org/10.7202/1077609ar

Aller au sommaire du numéro

Éditeur(s)

Alterstice

ISSN

1923-919X (numérique)

Découvrir la revue

Citer ce compte rendu

Dasen, P. R. (2011). Compte rendu de [Psychologies et cultures, sous la direction de B. Troadec et T. Bellaj]. Alterstice, 1(2), 129-132.

https://doi.org/10.7202/1077609ar d'utilisation que vous pouvez consulter en ligne.

https://apropos.erudit.org/fr/usagers/politique-dutilisation/ 


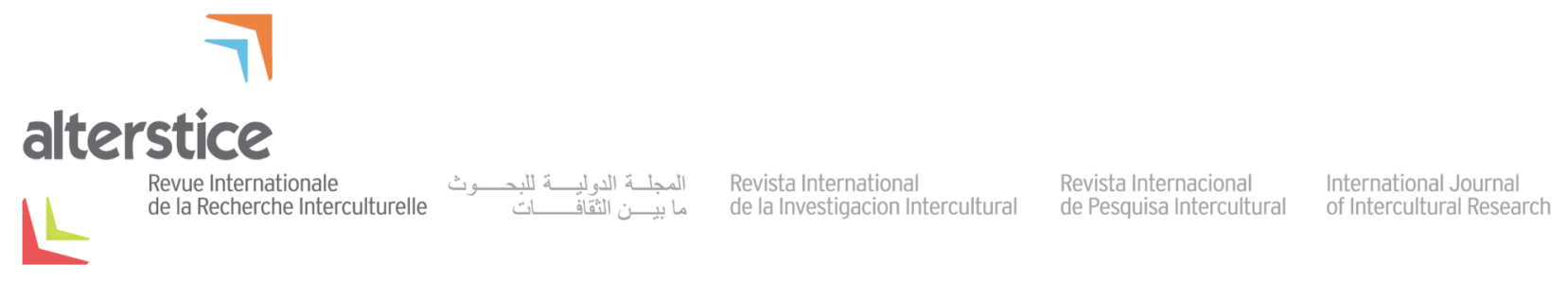

NOTE DE LECTURE

\title{
Psychologies et cultures, sous la direction de B.Troadec et T. Bellaj
}

Pierre R. Dasen ${ }^{1}$

\author{
Rattachement de l'auteur \\ ${ }^{1}$ Professeur honoraire, Université de Genève, Genève, Suisse \\ Correspondance \\ pierre.dasen@unige.ch \\ Références de l'ouvrage :
}

Troadec, B. et Bellaj, T. (dir.) (2011). Psychologies et cultures. Paris : L'Harmattan (Collection Espaces interculturels). (ISBN 978-2-296-55217-3)

\section{Pour citer cet article :}

Dasen, P. (2011). Psychologies et cultures [Note de lecture]. Alterstice, 1(2), 129-132.

\begin{abstract}
Voilà un livre que j'appelais de mes vœux depuis longtemps! Un livre exemplaire, qui fait le point sur les recherches les plus récentes en psychologie (inter-)culturelle comparée, et plus particulièrement sur les liens entre culture et cognition. Issu d'un symposium de l'Association de Psychologie Scientifique de Langue Française (APSLF) en collaboration avec la Société Tunisienne de Psychologie (STP), qui s'est tenu en Tunisie en septembre 2009, ce volume représente bien plus que de simples actes de colloque: il passe en revue de nombreuses questions très actuelles et pertinentes, et il est complété par quelques recherches empiriques originales. Les auteurs sont, d'une part, quelques collègues bien connus à l'Association internationale pour la Recherche InterCulturelle (ARIC) comme Colette Sabatier, Yvan Leanza et Bertrand Troadec lui-même, ou d'autres grandes pointures comme Patricia Greenfield ou Michel Fayol, et quelques collègues maghrébins comme Michèle Belajouza, Tarek Bellaj et Benaissa Zarhbouch, que cet ouvrage m'a permis de découvrir. Excellent choix, bien équilibré, qui illustre aussi ce que beaucoup d'entre nous recherchaient dans I'ARIC depuis longtemps, et qui s'était déjà réalisé lors du congrès à Alger : la possibilité d'apports et d'interactions entre collègues du Nord et du Sud.
\end{abstract}

Après une introduction par les deux éditeurs présentant l'ensemble du contenu, l'ouvrage s'ouvre avec une mise en perspective magistrale du domaine "culture et cognition ». Bertrand Troadec, tout à fait dans l'air du temps, convoque les théories de l'évolution aussi bien que les sciences cognitives. Dans une première partie, il utilise les spéculations de Mervin Donald, un neuropsychologue canadien, qui retrace les transformations de la cognition humaine au cours de la phylogenèse, en remontant à l'ancêtre commun aux primates qui aurait eu une culture épisodique, pour passer à l'homo erectus avec une culture mimétique, et l'homo sapiens, qui aurait commencé par une culture mythique puis serait passé à la culture scientifique sous l'influence de l'invention de l'écriture. 
Personellement, je me suis toujours méfié des ce genre d'évolutionnisme, qui a tendance à produire des « théories du grand partage " à la Horton $(1973,1991)$. Mais si je ne suis pas enthousiaste vis à vis de cette approche, je n'ai rien à redire sur le contenu. L'analyse est experte et très complète. Elle comprend un résumé circonstancié des travaux de Nisbett et de son équipe, et j'ai été satisfait de voir que Troadec présente le contraste entre pensée asiatique et occidentale non pas comme issu d'une différence de processus cognitifs, mais comme relevant de styles cognitifs. La conclusion est que si la cognition est un universel pour l'espèce humaine, son organisation dépend de mémoires externes (outils symboliques ou techniques), et que sa nature est donc à la fois neurale et contextuelle/culturelle.

Patricia Greenfield nous propose ensuite sa " théorie du changement social et du développement humain ", où elle fait explicitement le lien entre le changement historique et social et son effet sur le développement de l'enfant. Elle puise généralement ses exemples dans ses propres recherches longitudinales au Mexique et dans celles de sa collègue allemande Heidi Keller. Ce chapitre est la traduction d'un article déjà publié en anglais en 2009, et ne sera donc pas nouveau pour tout le monde, mais le fait qu'il soit maintenant disponible en français devrait inciter les lecteurs à s'intéresser aux travaux de ces deux chercheuses. Là encore, on peut s'offusquer de la simplification excessive de l'opposition communauté/société qui mènerait à collectivisme/individualisme, mais toute typologie est à la fois heuristique et frustrante. On peut regretter aussi que Troadec, au lieu de nous livrer une simple traduction, n'en ait pas profité pour éliminer les nombreux éléments redondants, et n'en ait pas fait un chapitre collaboratif, dans lequel il aurait pu introduire des liens avec la psychologie du développement de langue française, qu'il connaît si bien. (Je pense à cela par rapport à un exemple précédent de collaboration dans une traduction : Greenfield et Retschitzki, 1998.)

Dans le troisième chapitre, Michèle Belajouza traite des apprentissages, dont celui de la lecture, en particulier en fonction de la transparence de différentes langues au niveau orthographique: p. ex. en comparant l'arabe et I'hébreu, dont la transparence est simple, à l'anglais ou au français, où ce n'est pas le cas. L'auteure offre aussi une section intéressante sur la diglossie, notamment sur la coexistence de l'arabe dialectal et standard, puis elle introduit la question des apprentissages du système numéral dans différentes langues, thème qui est repris et développé dans le quatrième chapitre par Michel Fayol. En effet, ce dernier analyse toute une série de recherches récentes sur la construction du nombre, y compris la question de l'universalité de la "ligne numérique mentale » orientée de gauche à droite. II se trouve que cette question forme aussi le thème d'un numéro spécial récent du Journal of Cross-cultural Psychology (Göbel, Shaki et Fischer, 2011), dans lequel l'article le plus remarquable est celui de Rafael Núñez (2011), qui argumente contre cette universalité, soit en s'appuyant sur ses propres recherches récentes en Papouasie Nouvelle-Guinée, soit en réanalysant et corrigeant des résultats d'une recherche chez les Munduruku d'Amazonie qui semble avoir obtenu beaucoup d'attention dans la presse sur des bases assez peu fiables. Le chapitre de Fayol passe aussi en revue les recherches qui ont montré une supériorité des enfants asiatiques d'Extrême-Orient en mathématiques, reprenant l'hypothèse que ceci serait dû en partie à la transparence sus-mentionnée. L'auteur conclut (p. 168) :

Au total, les enfants américains, et plus généralement occidentaux, se voient handicapés par la forme phonologique des noms de nombres, qui contraint leur capacité de mémoire à court terme et, en cascade, leur apprentissage et mise en œuvre de stratégies de résolution des opérations [...]. La faible transparence de la base dix dans les systèmes numériques verbaux des langues occidentales influe négativement sur l'apprentissage de la numération écrite.

Ce chapitre complète fort bien une revue de la question plus ethno-pédagogique sur les " ethnomathématiques » que mes collègues et moi avions présenté au congrès de l'ARIC à Alger (Dasen, Gajardo et Ngeng, 2005).

Dans le chapitre suivant, Benaissa Zarhbouch nous dresse un tableau de la psychologie cognitive au Maroc, où l'auteur entrevoit le passage d'une psychologie au Maroc à une psychologie réellement marocaine. Après un aperçu historique, il nous présente quelques recherches menées en collaboration avec Bertrand Troadec, notamment une étude sur la flèche du temps, soit la représentation graphique classiquement linéaire avec le passé à gauche, le présent devant soi et le futur à droite. Sous l'influence de l'arabe qui s'écrit de droite à gauche, les élèves d'école primaire au Maroc trouvent dans les manuels des illustrations contradictoires ou mélangées ( $p$. ex. graphisme dans un sens, écriture dans l'autre). Les résultats de deux recherches empiriques montrent que des élèves bilingues marocains ou français (bilingues avec l'arabe ou une autre langue) utilisent effectivement

Alterstice - Revue Internationale de la Recherche Interculturelle, vol. 1, $n^{\circ} 2$ 
indifféremment les deux orientations dans leurs représentations graphiques, ce qui atteste une fois de plus de la flexibilité cognitive liée au bilinguisme, alors que des enfants monolingues suivent de façon plus rigide la norme culturelle, de droite à gauche pour les Français et de gauche à droite pour les Marocains. Ces derniers sont des enfants non scolarisés, ce qui montre que c'est bien une norme culturelle qui agit plutôt que l'apprentissage de la lecture en soi. Cette recherche illustre de façon exemplaire comment une recherche comparative quasiexpérimentale (c.-à.-d. où les groupes sont choisis selon des variables qui intéressent le chercheur, mais qu'on ne pourrait pas induire expérimentalement, comme le fait d'être scolarisé ou non) permet d'étudier des phénomènes qui restent inaccessibles à des recherches dans un seul contexte socio-culturel.

Les deux chapitres suivants ont une orientation de psychologie appliquée. Celui de Tarek Bellaj pose la question de l'adaptation des tests dans de nouveaux contextes culturels, question qui a fait l'objet de recommandations de la part de la Commission International des Tests (ITC). II est fort utile d'avoir un chapitre en français sur cette problématique (voir, en anglais, Hambleton et Zenisky, 2011). Quant à Yvan Leanza, il passe en revue les différentes façons de prendre en compte la culture dans les psychothérapies, ou de façon plus générale dans les consultations cliniques et dans la formation des professionnels de la santé. II retrace les problématiques principales et leur évolution dans les sphères anglophones et francophones, en y incluant une recherche bibliographique informatisée sur la dernière décennie. Celle-ci illustre qu'il y a, en fait, peu d'innovations récentes dans ce domaine, et qu'il vaut mieux avoir, comme Leanza, une vision plus large couvrant les trois dernières décennies ${ }^{1}$. L'auteur décrit tout d'abord les nombreuses tendances, par exemple les approches monographiques culturalistes qui dominent aux États-Unis, ou, dans le monde francophone, les avatars de l'ethnopsychiatrie. II en propose ensuite une typologie synthétique fondée sur l'intersection de deux dimensions, la connaissance de soi et de l'autre, par rapport à deux tendances extrêmes, la prescription ou au contraire l'exploration. II relève par ailleurs que le fait de prendre la culture au sérieux n'exclut plus de prendre aussi en compte les dimensions politique et économique, et que l'opposition entre universalisme et relativisme est maintenant dépassée. Il rejoint ainsi la conclusion de Troadec sur culture et cognition : "les humains sont semblables dans les profondeurs de leur psychisme, mais les particularités culturelles sont incontournables si l'on veut intervenir d'une façon qui prenne sens pour chacun d'eux » (p. 268).

Dans le chapitre final, Colette Sabatier revient sur les stratégies d'acculturation, à la suite des recherches de John Berry, mais en y ajoutant les pratiques parentales de socialisation et la dynamique familiale. Elle résume les résultats de ses propres recherches, l'une avec des adolescents et leurs parents en France et au Canada, une autre avec des adolescents marocains de seconde génération et leurs mères en France, ainsi qu'une grande étude comparative dans dix pays. Elle rapporte aussi les résultats d'une étude très originale et fascinante sur les styles identitaires et le choix de l'école en pays Basque, où les parents peuvent choisir entre des écoles bilingues et des Ikastola, écoles en immersion en langue basque, qui existent de façon illégale depuis 1969 et ont finalement été reconnues officiellement en 1999. C'est sans doute ce chapitre qui est le plus proche des intérêts de la plupart des membres de I'ARIC pour les questions relatives aux migrations, à la suite des travaux de Camilleri et collab. sur les "stratégies identitaires", mais il convient de noter que les recherches raportées par Sabatier ne portent pas exclusivement sur des migrants maghrébins en France.

C'est bien là, pour moi, un des grands intérêts de cet ouvrage : il illustre que les approches interculturelles sont pertinentes, qu'on étudie les questions de contacts entre cultures, au Nord comme au Sud, que cela soit dans le cadre des migrations ou des relations minorités (régionales)/majorités, et ceci en faisant des comparaisons interculturelles explicites ou non. De même, ces approches interculturelles sont pertinentes aussi bien pour des questions théoriques ou de science "fondamentale", comme dans l'étude de la cognition, que pour les préoccupations plus pressantes des praticiens en psychologie appliquée ou en santé mentale (à ce sujet, voir également Perregaux et collab., 2008, dans la même collection).

Bref, je disais en introduction que nous sommes en présence d'un ouvrage allant bien au-delà de classiques actes de colloque, et qui présente un panorama très complet et très actuel du thème " psychologies et cultures ", même s'il se focalise principalement sur la cognition. Comme il nous manque, en fait, un manuel en français de

\footnotetext{
1 Bien trop souvent, les recherches bibliographiques sont maintenant restreintes aux cinq ou dix dernières années, parce que ces outils informatiques incitent à ce choix. Or il y a des résultats de recherches qui restent valables pour bien plus longtemps que cela.
} 
psychologie inter-culturelle ce livre peut vraiment faire office de manuel de référence, un peu comme, en anglais, celui édité récemment par Van de Vijver et collab. (2011). Nous n'avons sinon que les deux ouvrages de Troadec $(1999,2007)$ lui-même, alors qu'il existe de nombreuses publications de ce type en anglais (p. ex. la troisième édition de Berry et collab., 2011). En tous cas, ce livre servira de base documentaire sérieuse pour les enseignants, surtout que le travail d'édition est bien fait, en particulier au niveau des bibliographies. Chaque chapitre peut ainsi représenter un modèle à suivre pour les étudiants.

Bravo et merci à Bertrand Troadec et à Tarek Bellaj pour cet excellent ajout à la collection «Espaces Interculturels », qui a pris une ampleur considérable depuis ses débuts héroïques, lorsque j'accompagnais Christiane Perregaux dans le lancement des premiers ouvrages.

\section{Références bibliographiques}

Berry, J., Poortinga, Y., Breugelmans, S., Chasiotis, A. et Sam, S. (2011). Cross-cultural psychology: Research and applications ( $3^{\mathrm{e}}$ edition revue). Cambridge : Cambridge University Press.

Dasen, P., Gajardo, A. et Ngeng, L. (2005). Éducation informelle, ethnomathématiques et processus d'apprentissage. Dans O. Maulini et C. Montandon (dir.), Formel? Informel? Les formes de l'éducation (p. 3963). Bruxelles : DeBoeck Université.

Göbel, S., Shaki, S. et Fischer, M. (2011). Cultural effects on the mental number line. Editorial to special issue. Journal of Cross-cultural Psychology, 42(4), 541-542.

Greenfield, P. et Retschitzki, J. (1998). L'enfant et les médias: les effets de la télévision, des jeux vidéo et des ordinateurs. Fribourg : Editions universitaires.

Hambleton, R. et Zenisky, A. (2011). Translating and adapting tests for cross-cultural assessments. Dans D. Matsumoto et F. J. R. van de Vijver (dir.), Cross-cultural research methods in psychology (p. 46-70). Cambridge : Cambridge University Press.

Horton, R. et Finnegan, R. (dir.). (1973). Modes of thought. London : Faber \& Faber.

Horton, R. (1991). La pensée traditionnelle africaine et la science occidentale. La pensée métisse. Cahiers de I'Institut Universitaire d'Etudes du Développement [Genève], 19, 45-67.

Núñez, R. (2011). No innate number line in the human brain. Journal of Cross-cultural Psychology, 42(4), 651-668.

Perregaux, C., Dasen, P., Leanza, Y. et Gorga, A. (dir.). (2008). L'interculturation des savoirs : entre pratiques et théories. Paris : L'Harmattan.

Troadec, B. (1999). Psychologie culturelle du développement. Paris : Armand Colin.

Troadec, B. (2007). Psychologie culturelle. Le développement cognitif est-il culturel? Paris : Belin.

Van de Vijver, F., Chasiotis, A. et Breugelmans, S. (dir.) (2011). Fundamental questions in cross-cultural psychology. Cambridge : Cambridge University Press. 